\title{
Publisher's Note: Interplay between tightly focused excitation and ballistic propagation of polariton condensates in a ZnO microcavity [Phys. Rev. B 92, 235308 (2015)]
}

\author{
R. Hahe, C. Brimont, P. Valvin, T. Guillet, F. Li, M. Leroux, J. Zuniga-Perez, X. Lafosse, G. Patriarche, and S. Bouchoule \\ (Received 20 January 2016; published 29 January 2016)
}

DOI: 10.1103/PhysRevB.93.039906

This paper was published online on 23 December 2015 with an error in a label in Fig. 8. Figure 8 has been replaced online as of 20 January 2016. The figure is incorrect in the printed version of the journal, therefore for the benefit of the print readership the figure has been replicated below.

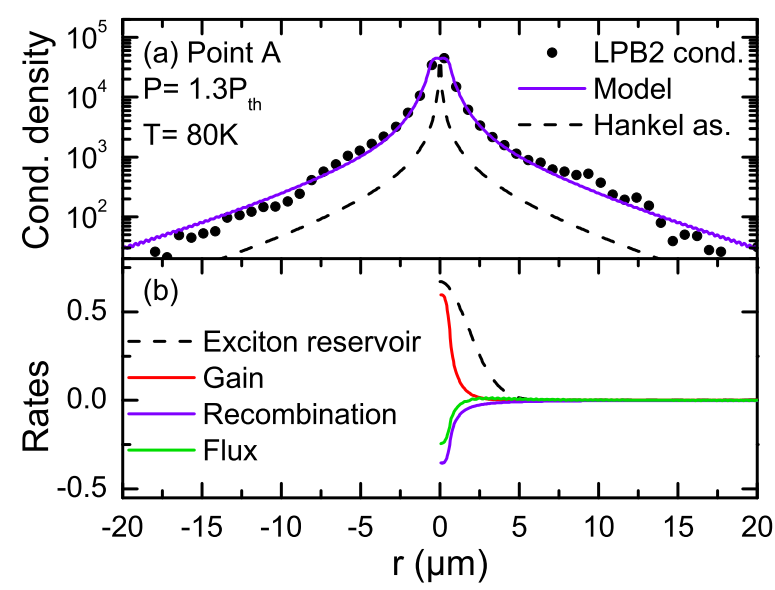

FIG. 8. (a) Experimental $y$-axis profiles of the condensate (dots) above the polariton laser threshold $\left(P=1.3 P_{\text {th }}, \mathrm{LPB} 2, T=80 \mathrm{~K}\right)$, and GPE simulation (plain line) and asymptotic Hankel function (dashed line); the blueshift of the condensate is $11 \mathrm{meV}$, as in the corresponding experiment (Fig. 5) and a reservoir density at the center is $710^{5} \mathrm{~cm}^{-2}$. The polariton decay rate $\gamma_{\mathrm{pol}}=0.4 \mathrm{meV}$. (b) Radial dependence of the simulated rates for gain (stimulated relaxation), polariton recombination, and polariton flux. 